\title{
A strategy for delivering high torsionality in longitudinal-torsional ultrasonic devices
}

\author{
Hassan Al-Budairi ${ }^{\mathrm{a}}$, Patrick Harkness ${ }^{\mathrm{b}}$, Margaret Lucas ${ }^{\mathrm{c}}$ \\ School of Engineering, University of Glasgow, Glasgow, G12 8QQ, UK \\ ah.al-budairi.1@ research.gla.ac.uk, ${ }^{\text {b Patrick.Harkness@glasgow.ac.uk, }}{ }^{\mathrm{c}}$ Margaret.Lucas@ glasgow.ac.uk
}

Keywords: ultrasonic, transducer, mode degeneration, longitudinal-torsional complex mode

\begin{abstract}
A composite longitudinal-torsional vibration mode has applications in ultrasonic motors, ultrasonic welding and ultrasonic drilling. There are two ways to obtain this vibration behaviour using a single transducer, namely (i) coupling of a longitudinal and a torsional mode, which is known to be difficult; and (ii) degenerating a longitudinal mode to deliver longitudinal-torsional behaviour at the horn tip. A mode-degenerating horn is achieved by incorporating helical or diagonal slits in an otherwise traditional exponential horn driven by a Langevin transducer. However, it is often difficult with this configuration to avoid coupling of unwanted bending modes, low responsiveness, and loss of ultrasonic energy due to boundaries between tuned components. Therefore, in this study the mode-degenerating characteristics are achieved by incorporating the helical slits and exponential geometry features in the front mass of the transducer itself. Finite element analysis and vibration experimental analysis show that this strategy prevents coupling of bending modes, increases responsiveness, and reduces energy losses. Most importantly the transducer delivers a very high torsionality.
\end{abstract}

\section{Introduction}

Use of the composite longitudinal-torsional vibration mode in ultrasonic systems has become commonplace, and applications such as ultrasonic motors, ultrasonic welding and ultrasonic drilling have benefitted from complex vibration patterns achieved through specialist horns.

The generation of composite longitudinal-torsional vibration modes in ultrasonic systems consisting of a single transducer, with or without an acoustic amplifier (booster) and horn, is generally achieved by making a geometric modification such that a generated longitudinal mode can deliver energy to the composite vibration mode. Although energy conversion efficiency may be low, this method is preferred due to its simplicity of both manufacture and use. The main alternative, namely 
using two sets of transducers to generate the two specific modes, is known to be difficult and so is a relatively expensive approach [1].

There are two different techniques by which geometric modification can be used to achieve a composite mode. The first method is coupling a longitudinal and torsional mode so that they resonate exactly at the same frequency, as illustrated by Lin [2], who performed a theoretical and experimental study on coupling in an exponential horn. To couple longitudinal and a torsional modes the speed of sound should be the same in both, and in order to reach this condition careful selection of a decay constant of the cross section area of an exponential solid horn should be made. However this may produce horns which are long but small-tipped, and therefore poorly-suited to many ultrasonic applications.

The second method is degeneration of a single longitudinal mode in order to deliver a composite longitudinal-torsional output at the tip of the horn. Significant work has been done to increase the torsionality of these horns, where torsionality is the ratio of torsional to longitudinal response amplitude at the circumference of the horn tip. Harkness [3] has suggested that a helical flute in the thinner stem section of a stepped horn could increase the torsionality, but these horns appear to be especially vulnerable to unwanted bending modes.

In this paper, a new method is suggested to degenerate the longitudinal vibration mode of an ultrasonic transducer to produce a longitudinal-torsional composite mode in the transducer itself. Geometric modifications in the front mass of the transducer, including exponential cross-sectional area reduction and slotting, are used to increase the torsionality ratio, prevent the appearance of unwanted bending modes, and lessen ultrasonic energy losses through reduction of inter-component boundaries.

\section{Principle of mode conversion using slots}

The use of slots to convert longitudinal vibration into a composite longitudinal-torsional behaviour has been explained by Tsujino [4]. For a cylindrical wave guide, cutting shallow slots about the circumference such that the inner part remains unslitted will led to conversion of the incident longitudinal wave from a vibration source on the input side to a torsional wave at the diagonal slits, whilst the remainder of the wave progresses unaltered through the unslitted core. These principles have been employed in the conical front mass of a modelled transducer as shown in Fig.1, where the slots have been cut partially through the mass and rotated around the longitudinal axis (zdirection) of the transducer. 


\section{Finite element model development}

Multiple transducer models have been created and analysed in ABAQUS finite element software to evolve the maximum torsionality ratio. Fig.1 shows a diagram of the final transducer which consists of a stainless steel back mass, PZT-8 piezoceramics with copper electrodes, a titanium central prestress bolt, and a partially-slotted titanium front mass. Transducer materials are chosen such that the acoustic impedances of the front and back masses match that of piezoelectric material and, therefore, minimise internal reflections [5]. In order to avoid unnecessarily complicated models of the joint interfaces and piezoelectric losses, a global damping ratio is used for all materials. The value is found using the Rayleigh formula, the viscous constant $\beta$ being defined from the following expression:

$$
\beta=\frac{1}{\omega_{r} \cdot Q_{m}}
$$

In this expression $\omega_{r}$ is the resonance frequency of the vibration mode being analysed, and $Q_{m}$ is a mechanical quality factor which is estimated at the beginning of the analysis and then corrected after an experimental harmonic analysis of the fabricated transducer.

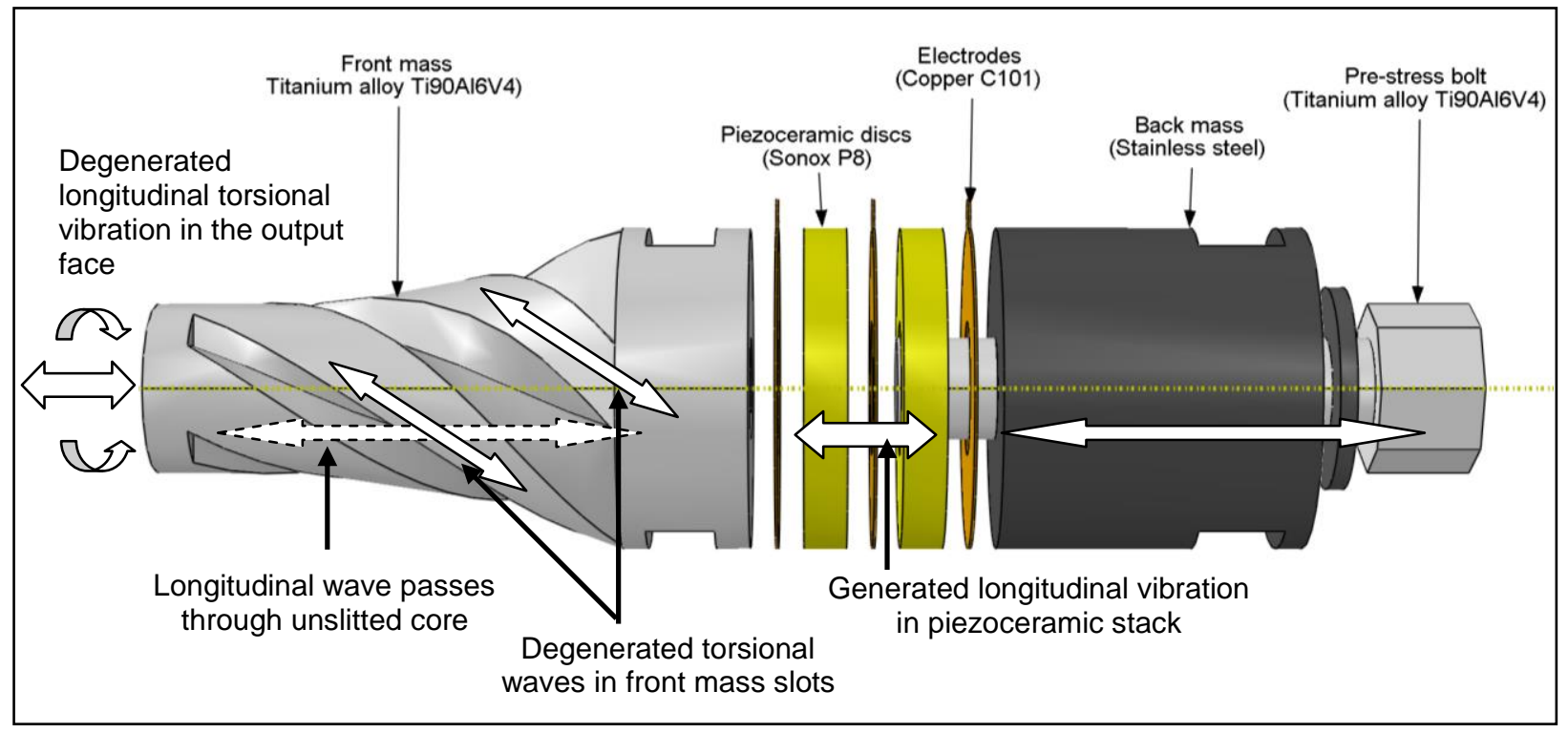

Fig. 1. Schematic of piezoelectric transducer and degeneration of vibrational modes.

\section{Experimental analysis and results}

Fig. 2 shows the fabricated piezoelectric transducer. In order to study the longitudinal and torsional responses along the transducer, 18 points have been selected on transducer along the z-direction and the responses in $\mathrm{x}$-direction and z-direction have been extracted as shown in Fig.3. The same process is carried out for numerical model in order to compare the results. The experimental modal analysis (EMA) and numerical finite element analysis (FEA) results are shown in Fig. 4. The EMA is carried out by exciting the transducer with a random signal over a broadband frequency range (0- 
$80 \mathrm{kHz}$ ) whilst the response is measured using 3D laser Doppler vibrometer. The experimental results show that the desired mode is resonates at $19,036 \mathrm{~Hz}$ and the surrounding vibration modes are a bending mode (at 15,706 Hz) and a torsional mode (at 22,077 Hz). These results are in good agreement with the FEA results (less than 6\% difference) and the slight differences may be ascribed to errors in the accuracy of material specifications, the boundary conditions, and the linearity analysis condition of the software. It is apparent that there is a good separation, more than $15 \%$, between the frequency of the desired degenerated vibration mode and that of the surrounding unwanted bending modes.

Fig. 5 shows the experimental longitudinal and torsional responses to a range of continuous swept sine excitations. The response is measured at a point on circumference of the transducer tip. Variation of torsionality with the excitation frequency is shown in Fig. 5 and Fig. 6 shows the numerical and experimental peak responses at resonance and the corresponding torsionality ratio versus the excitation voltage. It is found that the torsionality is slightly decreases with excitation frequency. Also it is apparent that the torsionality is not a strong function of the excitation voltage, a finding which is consistent with the numerical analysis. There is also a slight softening effect due to the nonlinear behaviour of piezoceramic material.

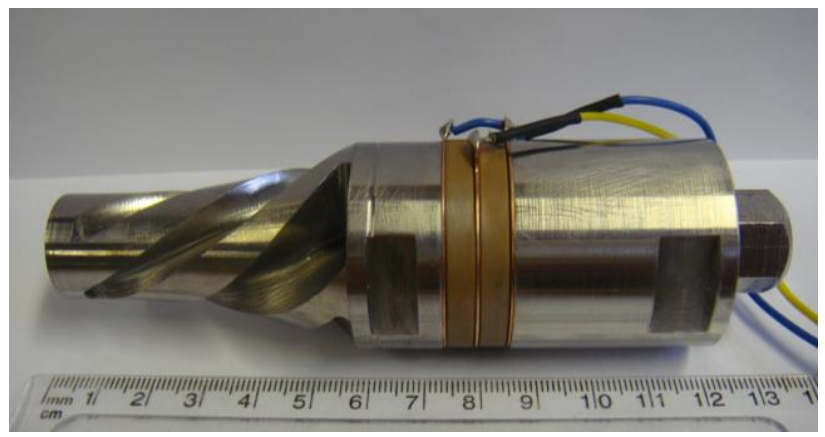

Fig. 2. Fabricated transducer.

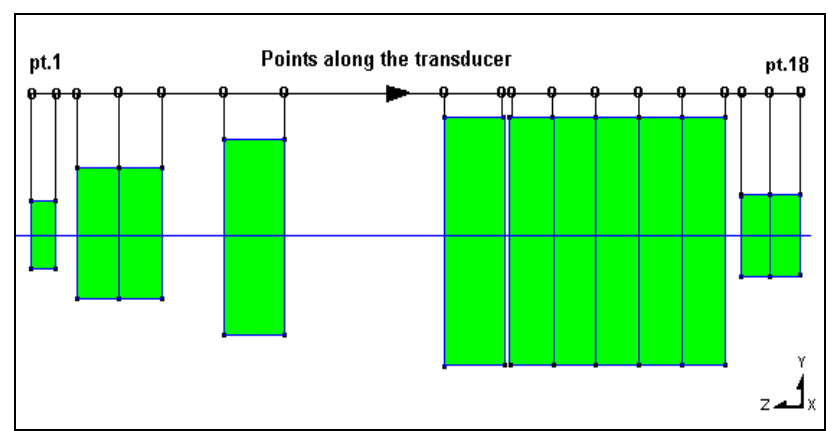

Fig. 3. Representation of transducer in EMA. 


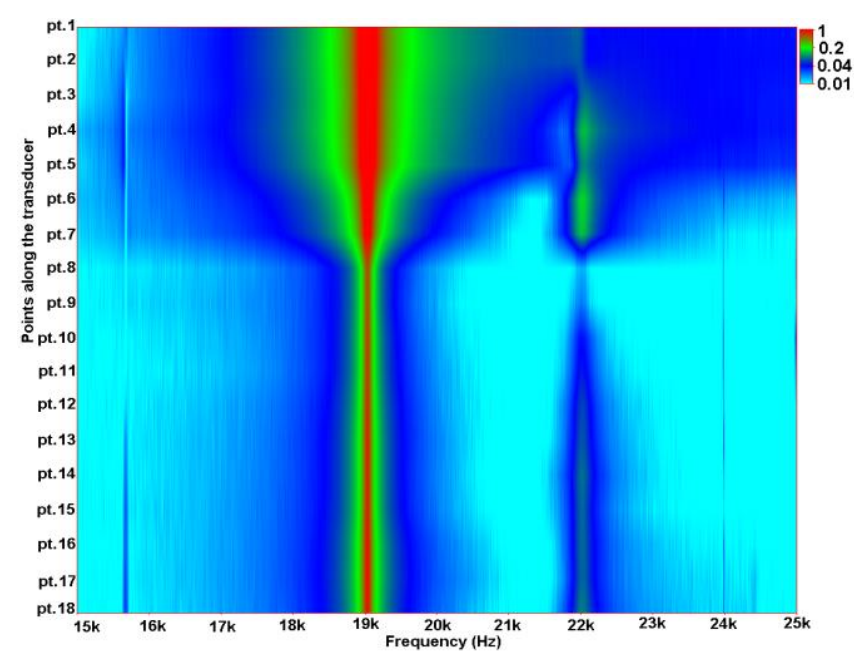

(a) EMA longitudinal response.

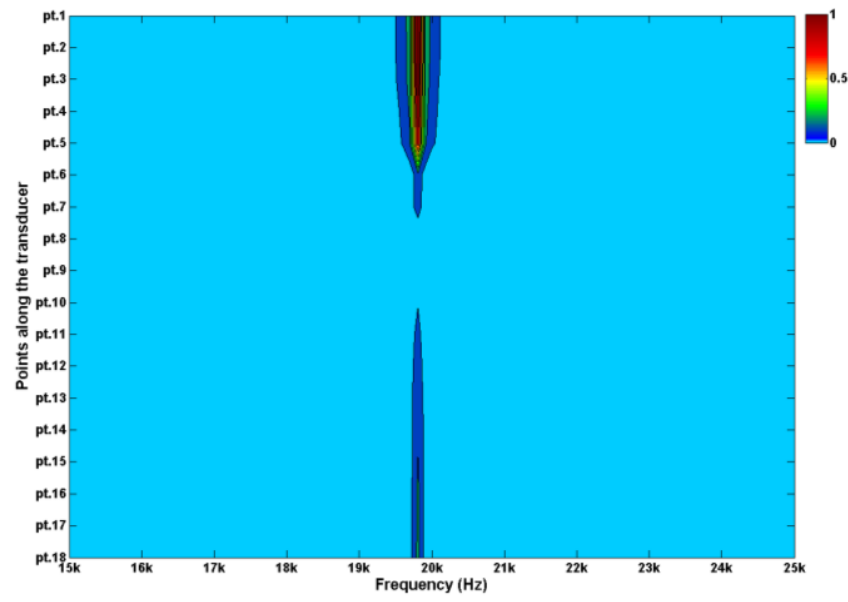

(c) FEA longitudinal response.

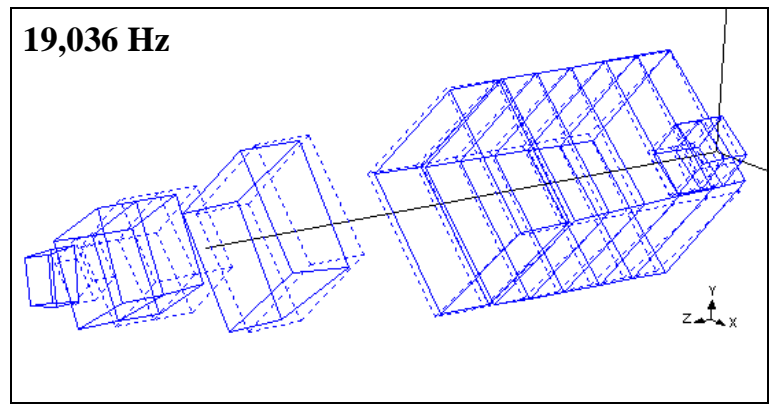

(e) Mode shape of degeneration vibration mode.

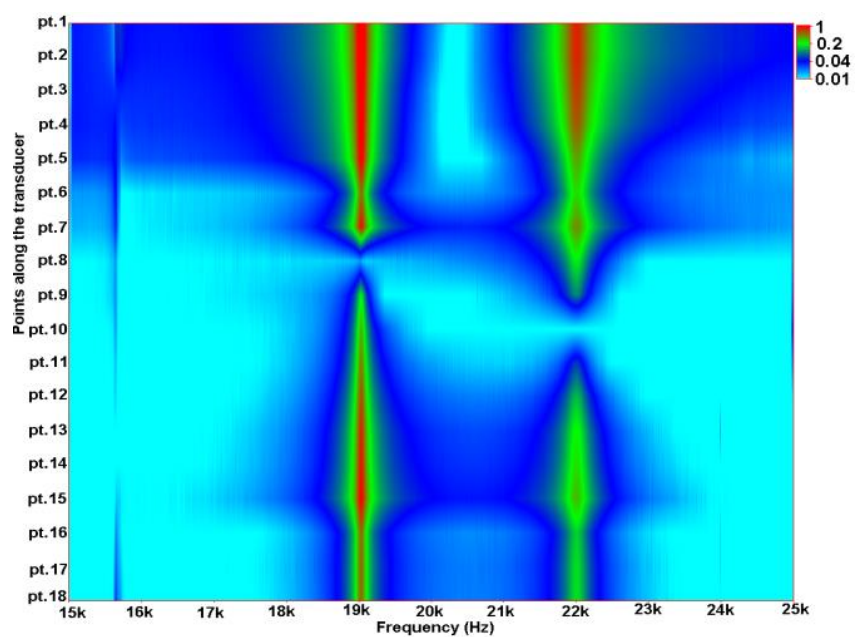

(b) EMA torsional response.

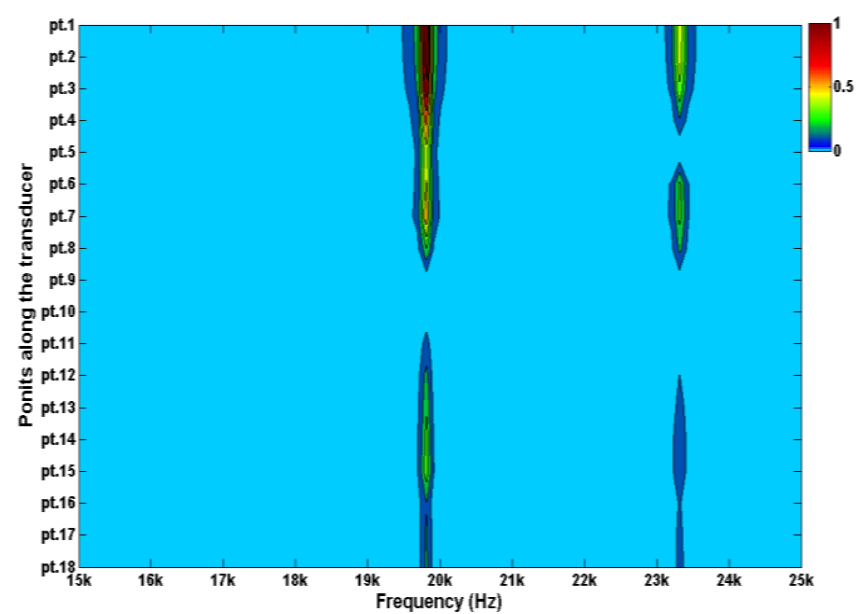

(d) FEA torsional response.

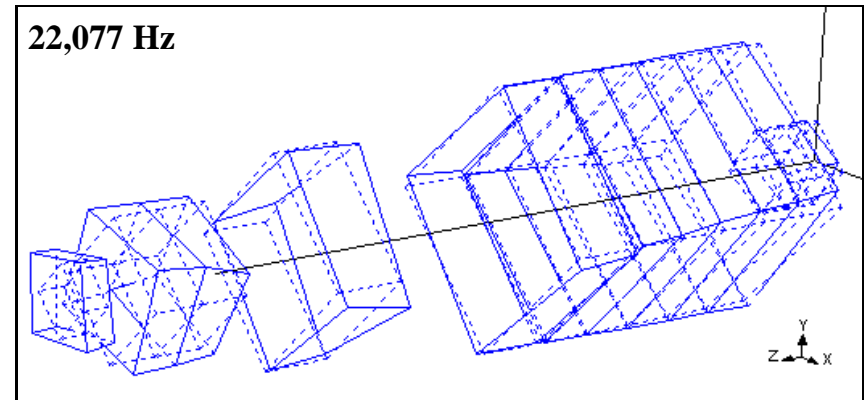

(f) Mode shape of surrounding torsional mode.

Fig. 4. Experimental and numerical modal analysis with mode shapes of desired and surrounding modes of vibration.

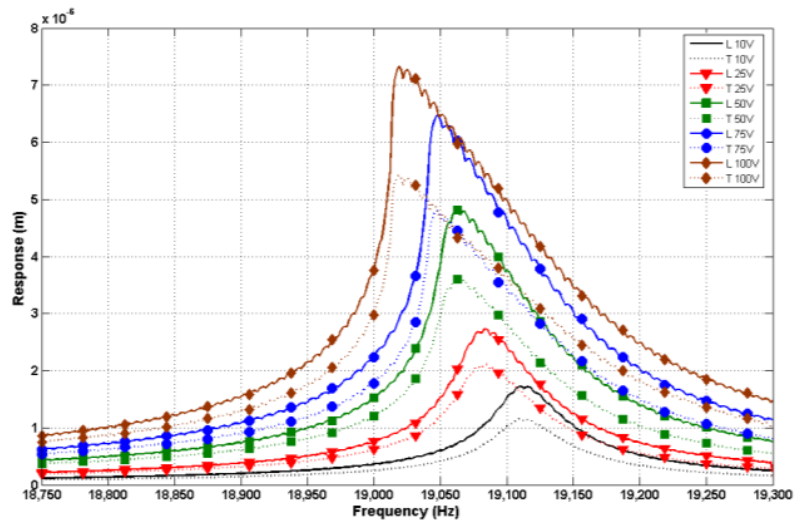

Fig. 5. (left) Longitudinal (L) and torsional ( $T$ ) responses of different excitation voltages. 


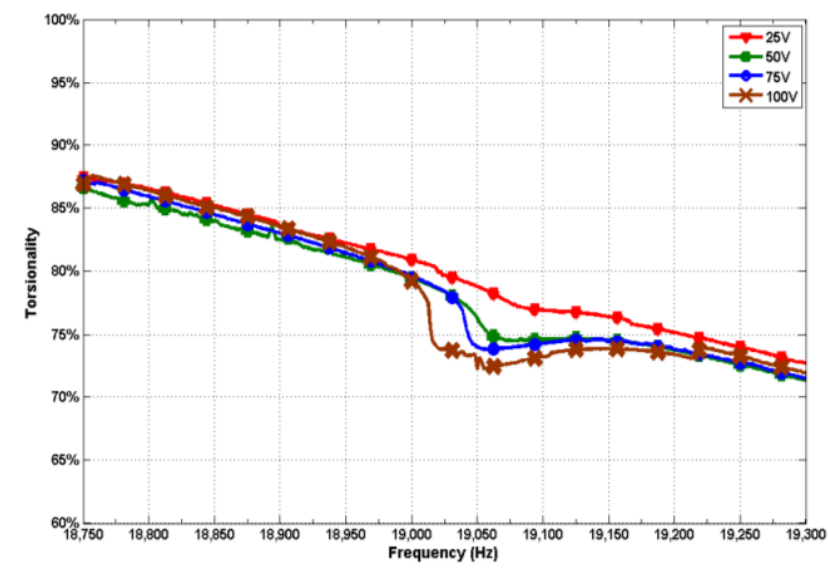

Fig. 6. Change of torsionality ratio with excitation frequency for different excitation voltages.

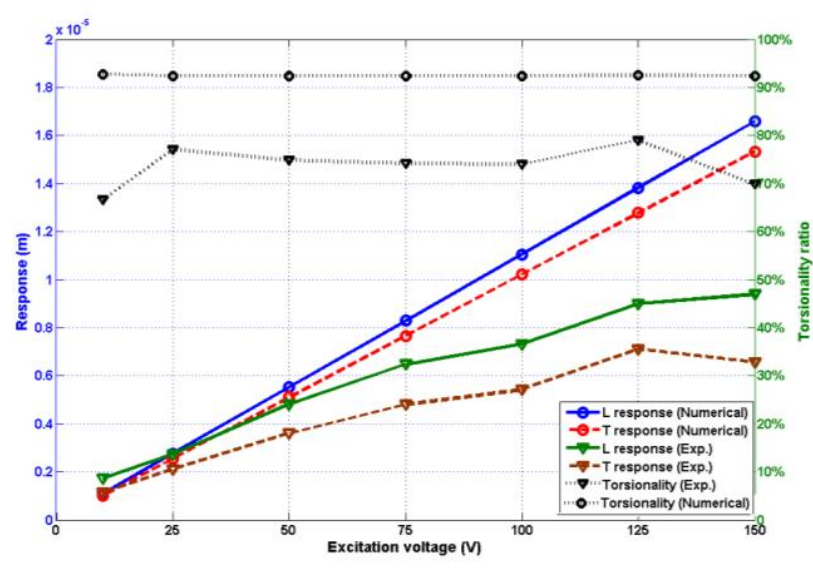

Fig. 7. Longitudinal (L) and torsional (T) peaks and torsionality ratio for different excitation voltages.

\section{Conclusion}

Modifications to the front mass of piezoceramic transducer were designed using finite element software, the optimum design being manufactured and analysed to determine how effectively it degenerates an excited longitudinal mode into a composite longitudinal-torsional behaviour at the output face of the transducer. The combination of exponential area reduction and helical slotting in the front mass is shown to be an effective technique, delivering high responsiveness and torsionality without bending modes interfering with the desired output. The simplicity of the manufacture and excitation of the device makes it practical for use in a wide range of applications requiring some degree of longitudinal-torsional vibration output.

\section{References}

[1] L.D. Rozenberg: Sources of High-Intensity Ultrasound, Vol. 2 (Plenum Press, New York, USA, 1969).

[2] S. Lin: Study on the Longitudinal-torsional Composite Mode Exponential Ultrasonic Horns, Ultrasonics, Vol. 34(7), (1996), p. 757-762.

[3] P. Harkness, A. Cardoni and M. Lucas: Ultrasonic Rock Drilling Devices Using Longitudinal-Torsional Compound Vibration, (2009 IEEE International Ultrasonics Symposium, Rome, Italy 2009).

[4] J. Tsujino: Ultrasonic Complex Vibration Systems and Various Applications of High Power Ultrasonics, (European and Japanese Symposium on Acoustics, Sevilla, Spain, 2002).

[5] R. Mahdavinejad: Finite Element Dimensional Design and Modeling of an Ultrasonic Transducer, Iranian Journal of Science \& Technology, Transaction B, Engineering, 29(B2), (2005). 